551. 509. 33: 551.543 .3

\title{
Studies on the Seasonal Weather Forecasting (II)
}

- Periodicities in Meteorological Phenomena-

by

K. Takahashi and Collaborators

Meteorological Research Insitute

(Received February 5, 1952)

\begin{abstract}
Predominating periods of daily pressures at several stations in Japan were investigated by harmonic analysis. We found 5-, 12 13-day and probably 4- and 10-day periods predominated throughout the year. Analysing 5-day mean values of pressure and temperature deviations from the normal at some stations over Asia and the North Pacific, we found 25-, 35-, 45-, and 65-day periods predominated. The latter long periods coincide with the values which were analysed formerly by so-called periodogram analysis. 5- and 9-month periods were also found to predominate. Assuming such a long period, 5-day mean pressure at the stations over the Northern Hemisphere was analysed by harmonic analysis, and we found that they were expressed approximately by a regular standing oscillation of pressure over the earth. Next, 8-day period of pressure over: the Northern Hemisphere was analysed by similar methods, and such an oscillation was suggested to be an internal wave of atmosphere.

Among many short periods, 4-day period at $500 \mathrm{mb}$ level over the Northern Hemisphere was investigated in detail. 8 or 9 planetary waves round the Northern Hemisphere seem to be the origin of the 4-day period. Heights of $500 \mathrm{mb}$ level along latitude $50^{\circ} \mathrm{N}$ in winter were analysed into elementary sinusoidal waves by harmonic analysis and two kinds of waves, one a stationary and the other a propagating wave, were found. It was found that long waves propagate towards the west and short waves towards the east. Thus, modes of the oscillation of long periods and short periods are quite different.

Some trials of long range forecasting were made, assuming such periodicities. Analysing the pressure variations of several stations to the elementary oscillation and extrapolating such elementary oscillation to the future and adding them, the forecasted values of pressure are obtained.
\end{abstract}




\section{Method of analysis}

In our previous report, periodicities of 5-day mean temperature and pressure were analysed by SCHUSTER's periodogram analysis. However, such a method is not convenient for unstable oscillations such as seiches of lakes. In this report, harmonic analysis will be applied. We will explain briefly the method of analysis, though the harmonic analysis are explained in various textbooks.

Let time series of any value, such as temperatures and pressures be expressed approximately as

$$
f(t)=\sum_{i}\left(a_{i} \cos 2 \pi \nu_{i} t+b_{i} \sin 2 \pi \nu_{i} t\right)
$$

Multipling ( 1 ) by $\sin 2 \pi \nu_{0}$ and $\cos 2 \pi \nu_{0}$ respectively, and integrating it for time interval from 0 to $n / \nu_{0}$ i. e., $n$ times of the trial period $1 / \nu_{0}$ and lastly subdividing it by $n / 2 \nu_{0}$, we get

$$
\text { (2) }\left\{\begin{array}{l}
\mathrm{A}=\frac{2 \nu_{0}}{n} \int_{0}^{n / \nu_{0}} f(t) \cos 2 \pi \nu_{0} t d t=\sum_{i} \frac{\nu_{0} \nu_{i}}{n \pi\left(\nu_{0}^{2}-\nu_{i}^{2}\right)}\left\{-a_{i} \sin 2 n \pi \frac{\nu_{i}}{\nu_{0}}-b_{i}\left(1-\cos 2 n \pi \frac{\nu_{i}}{\nu_{0}}\right)\right\} \\
B=\frac{2 \nu_{0}}{n} \int_{0}^{n / \nu_{0}} f(t) \sin 2 \pi \nu_{0} t d t=\sum_{i} \frac{\nu_{0}^{2}}{\left.n \pi \cdot \nu_{0}^{2}-\nu_{i}^{2}\right)}\left\{a_{i}\left(1-\cos 2 n \pi \frac{\nu_{i}}{\nu_{0}}\right)-b_{i} \sin 2 n \pi \frac{\nu_{i}}{\nu_{0}}\right\}
\end{array}\right.
$$

When $\nu_{0}$ is equal to $\nu_{i}$ or nearly so,

$$
A=a_{i}, \quad B=b_{i}
$$

and hence $C=\sqrt{A^{2}+B^{2}}$ is equal to the amplitude of the wave contained in $f(t)$. When $\nu_{0} \neq \nu_{i}, A$ and $B$ are small. This is the principle of harmonic analysis. Though unstable oscillations can not be analysed by harmonic analysis, usual oscillations may be considered to be almost stationary for a short time interval: Therefore, dividing time series of observed data into several time intervals equal to $n$ times of the trial period $1 / \nu_{0}$ and applying harmonic analysis to every time interval, and then calculating the mean amplitude, we can find the predominating periods. The square mean of these amplitudes is calculated as

$$
\sqrt{\overline{C^{2}}}=\frac{2 \nu_{0}}{n \pi} \sqrt{\frac{\sum_{i} \overline{a_{i}^{2}}\left(\nu_{0}^{2}+\nu_{i}^{2}\right) \sin ^{2} n \pi \frac{\nu_{i}}{\nu_{0}}}{\left(\nu_{0}^{2}-\nu_{i}^{2}\right)^{2}}}
$$

under the probable assumptions that

$$
\overline{a_{i}^{2}}=\overline{b_{i}^{2}}, \quad \overline{a_{i} b_{j}}=0 ; \quad \text { if } \quad i \neq j, \quad \overline{a_{i} a_{j}}=0, \quad \overline{b_{i} b_{j}}=0 .
$$

This is the principle of the present method.

When $f(t)$ contains only one period, (4) becomes

$$
\sqrt{\overline{C^{2}}}=\frac{2 \nu_{0}}{n \pi} \frac{\sqrt{\overline{a_{i}^{2}}} \sqrt{\nu_{0}^{2}+\nu_{i}^{2}}}{\nu_{0}^{2}-\nu_{i}^{2}} \sin n \pi \frac{\nu_{i}}{\nu_{0}}
$$

for the mean amplitude of the wave is $\sqrt{a_{i}^{2}+b_{i}^{2}}=\sqrt{2 a_{i}^{2}}$, (6) is rewritten as

$$
\sqrt{\overline{C^{2}}}=\gamma \sqrt{2 a^{2}}
$$

where the magnifing factor $\gamma$ is

$$
\gamma=\frac{\sqrt{2}}{n \pi} \frac{\sqrt{1+K^{2}}}{\sqrt{1-K^{2}}} \sin n \pi K
$$




$$
K=\nu_{i} / \nu_{0} \text {. }
$$

Thus, factor $\gamma$ depends on the trial frequency $\nu_{0}$ and $n$. In Fig. 1, the relations between $\gamma$ and $K$ for $n=1,2,3, \cdots \cdots$ are shown.

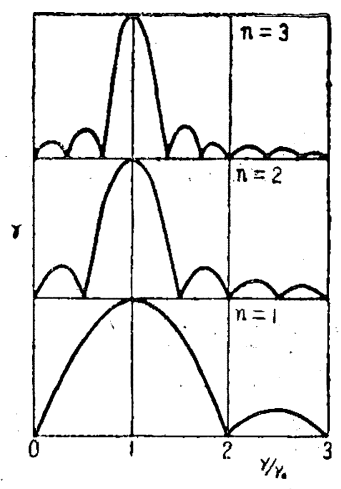

Fig. 1.

Relation between $r$ and $K$

Accordingly, if we calculate the amplitude from observed data by the present method, taking various trial frequency $\nu_{0}$, the amplitude of the oscillation in the original data will become largest when $\nu_{0}=\nu_{i}$, or, in other words, the spectrum of the analysed amplitudes for $\nu_{0}$ will represent roughly the spectrum of the original oscillations, and the hidden periodicity will be detected.

The resolving power of the present method depends on the duration of the integral, or $n$, and it is calculated roughly as follows under some plausible assumptions.

Thus, when $n=3$, the difference $21 \%$ of the period is resolved. Accordingly, taking $n$ large, the resolved power becomes large. However, when $n$ is large, the waves are not stable and the assumption of the present method holds no more. Thus the selection of $n$ depends on the character of the time series.

It must be noticed further, that the spectrum of the analysed amplitude has a certain value even if $f(t)$ are random. When time series are random, (6) becomes,

$$
\sqrt{\overline{C^{2}}}=2 \sqrt{\frac{\nu_{0}}{n} \sigma_{f}}
$$

where $\sigma_{i}$ is the standard deviation of $f(t)$ and the standard errors of (10) are caluculated to be

$$
\delta \sqrt{\overline{C^{2}}}=\sigma_{f} / \sqrt{ } 2 j
$$

where $j$ is the number of data subjected to harmonic analysis.

\section{Resluts of analysis}

Y. ARAI and M. HIROSE analysed daily pressures at several stations in Japan $1934 \sim 1936$ by the above methods, taking $n=3$. One of the analysed spectra for winter, spring, summer and fall is shown in Fig. 2. We found that some predorninating periods exist in pressure variations and they are almost the same throughout the year. The values of such period are 5-, 8-, 12

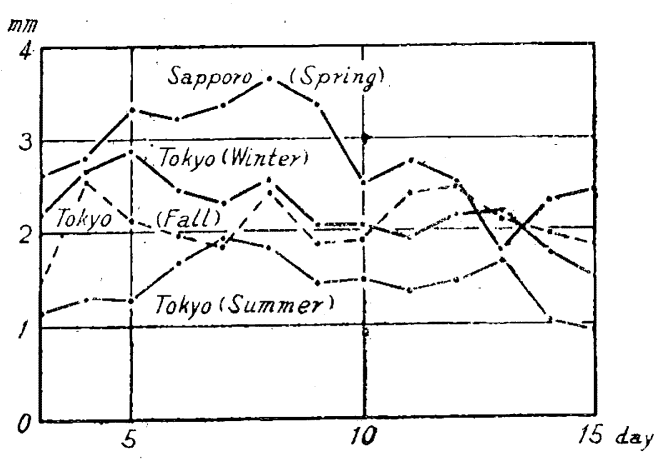

Fig. 2. Spectra of several days period. (Anomaly of Pressure) 
13-days and probably 4 and 10-days. We found also that daily mean temperature has similar predominating periods.

K. TAKAHASHI analysed 5-day mean pressure at several stations in Asia and

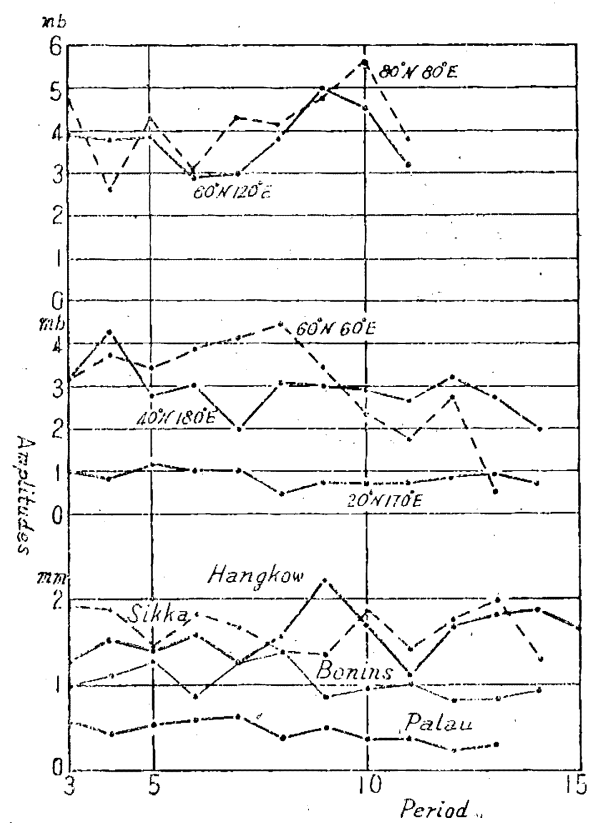

Fig. 3. Spectra of 5-day mean pressure. Period (Unit $=5$ days) the Pacific, some of the analysed spectra are shown in Fig. 3. Glancing at such spectra, we found 25-, 35-, 45 and 65-day periods predominatr. Such values of periods coincide with the values obtained by periodogram analysis in our previous report [1].

WEICKMAN [2] and his successor [3] found 24- and 36-day periods in Europe and BRoOKs [4] noticed that 12 13-, 18 19-, 26-, 48- and 72-day periods predominate. These periods agree with ours at least in first approximation, and hence we may consider that these periods predominate over the whole earth.

$\mathrm{K}$. TAKAHASHI analysed also the deviations of monthly mean temperature from the normal at several stations in Japan and 9- and 5-month periodicities were found to predominate.

\section{Analysis of long periodic oscillations}

T. ANDO and N. NAKAMURA investigated the mode of 25-, 35-, 45- and 65-day periods over the Northern Hemisphere. Using 5-day mean pressure at stations of every $10^{\circ}$ of latitude and $20^{\circ}$ of longitude, they calculated the phase angles and amplitudes of such oscillations by harmonic analysis. Namely, on the assumption that pressure variations were expressed by

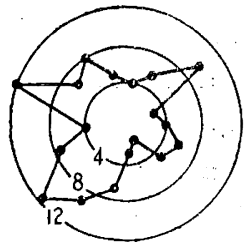

$T=25$

$$
F_{i}(t)=A_{i} \sin \left(2 \pi \frac{t}{T}+\varphi_{i}\right)
$$

$A_{i}$ and $\varphi_{i}$ at every station were calculated from the observed data of any 3 months of 1949 and 1950. After such calculations, we found the following interesting characters.

Frequency distributions of phase angles over the Northern Hemisphere for periods

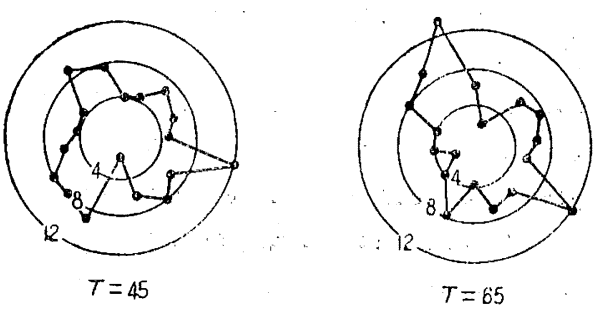

Fig. 4. Frequency distributions of phase angles of $25 \cdots, 35$, 45 and 65-day pericd. 
of 25-, 35-, 45-, and 65-day are shown in Fig. 4. Glancing at these, we know that the phase angles of the oscillations over the Northern Hemispherè may be divided into two groups and the phase difference of the two is about $180^{\circ}$. This means that such pressure oscillations over the Nor thern Hemisphere can not be considered as progressive but standing.

In Fig. 5 the distributions of the phase angles and amplitudes over the Northern Hemisphere are shown. Glancing at these figures, we know that phase angles take various values where the amplitudes are comparatively small and similar values where the amplitudes are comparatively large. This is also explained by assuming that the oscillations are standing. Of course, such oscillations take different patterns for different periods. Next, we will explain briefly the pattern of each oscillation.

The pattern of the oscillation of 65-day period suggests that the oscillation is half-hemispheric and such a pattern of the oscillation is almost similar for any season and year.

The pattern of the oscillation of 45-day period is expressed roughly by a concentric belt which centered at the pole. When pressure near the pole and the lower latitudes rises, pressure in the middle latitude falls.

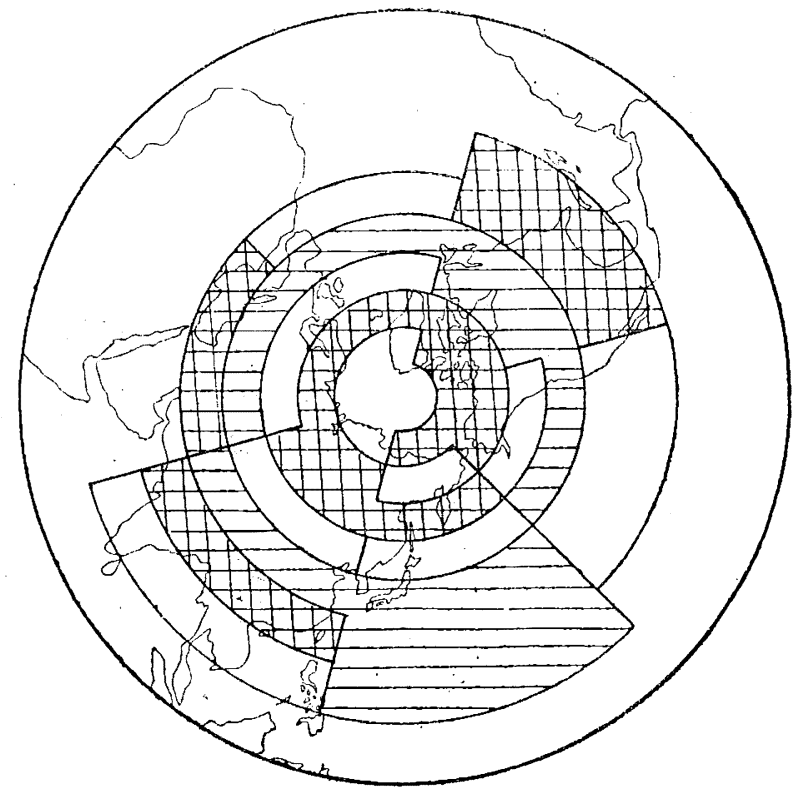

Fig. 6. a. Distribution of the phase angle of 45-day period. (Zonal index at"suface, in winter) Phase angle is opposite between hatched area and cross hatched area.

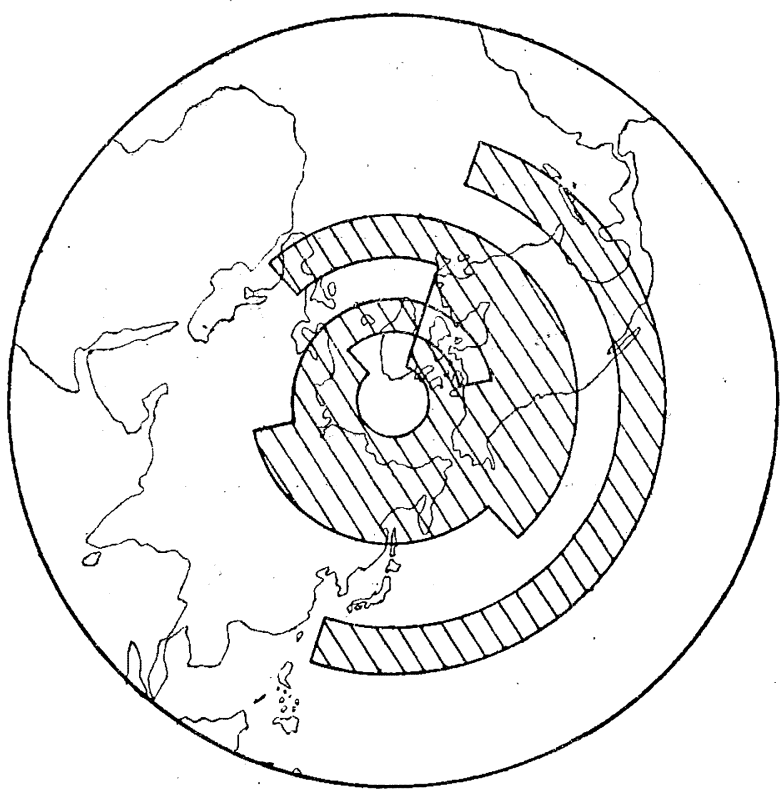

Fig. 6. b. Distribution of the amplitude of 45-day period. (Zonal index at surface, in winter) Hatched areas indicate large amplitude areas.

The pattern of 35-day period suggests that they are the oscillation of the action centers such as the Siberian high and the North American high. Thus, 
when the Siberian high and middle high develop, the frontal zone between them develops, hence the phase angle over Siberia and that near Japan are opposite.

25-day period is not so simple as the other periods. It seems to be caused by the oscillation between pressures at high and low latitudes. The pattern of the oscillation in summer and winter are not quite similar, but do not differ for every year.

M. OKUTA analysed the zonal index between the mean pressures at every $10^{\circ}$ in latitude by similar method. In this case, to make the above mean values we used the six estimated values at every $60^{\circ}$ of longitude. In Fig. 6, one of his results is shown. We see that the pattern of 45-day period is quite similar with the zonal

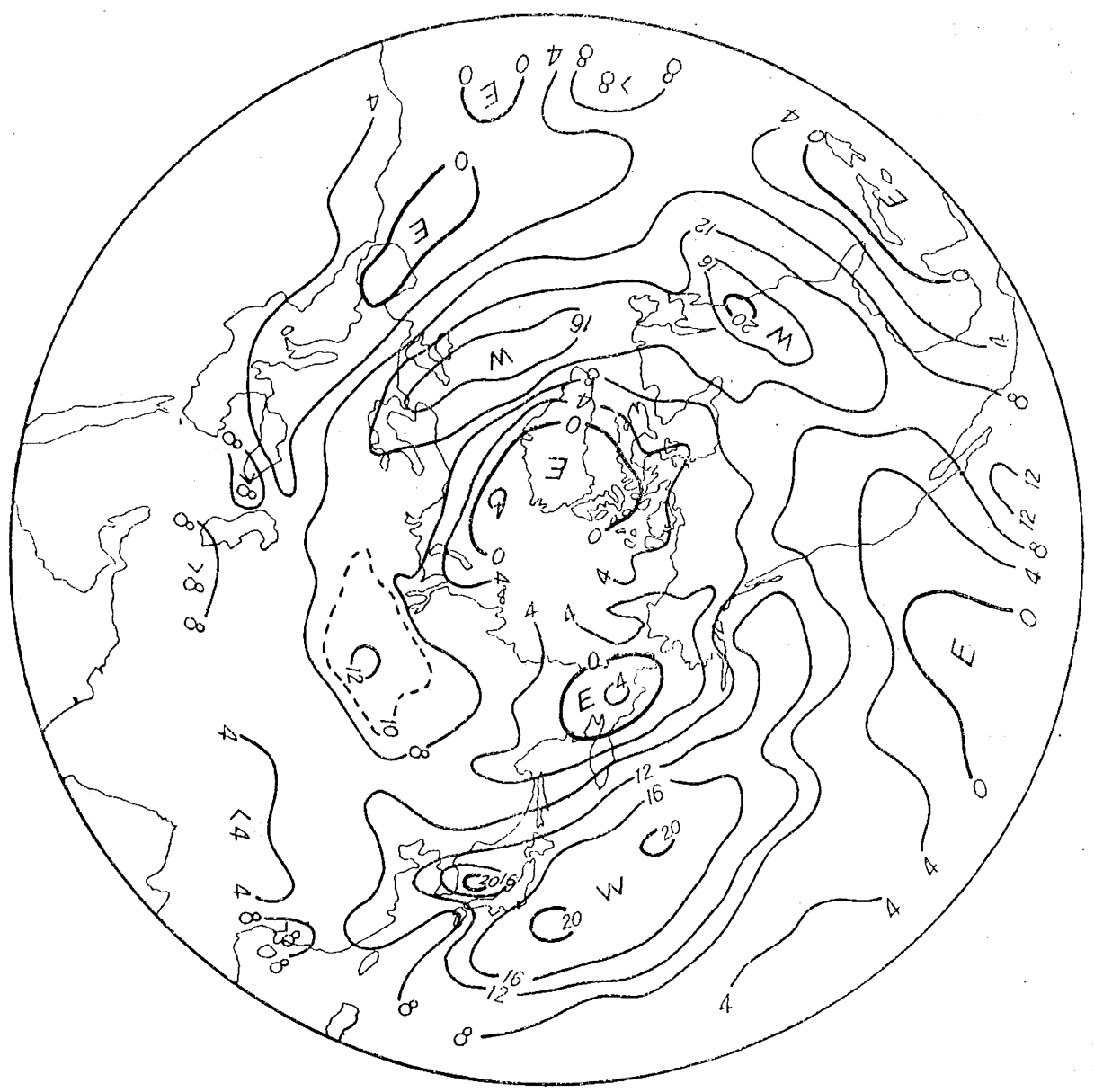

Fig. 7. Distribution of zonal flows at $700 \mathrm{mb}$ level in Jan. 1949.

flow at $700 \mathrm{mb}$ level which is shown in Fig. 7. This suggests that 45-day period is the oscillation of zonal flow, and when polar and trade easterlies intensify, the middle latitude westerlies weaken.

Though the apparent patterns of ANDO's and OKU'TA's analysis seem to be 
somewhat different, they are the same oscillation, and we may conclude that such a long periodic oscillation is a phenomenon of worldwide scale, probably the standing oscillation of the atmosphere. The mechanism of such an oscillation will be discussed in a future report.

\section{8-day periodicity in weather changes}

7-day periodicity is one of the famous periodicities in weather changes. Y. ARAI analysed such a periodicity by similar harmonic analysis in a previous section. Assuming 8-day period instead of 7-day, and using $500 \mathrm{mb}$ height over the Northern Hemisphere, during 1950, he obtained the distribution of the amplitudes and the phase angles of 8-day period over the Northern Hemisphere as shown in Figs. 8 and 9, and the frequency distribution of the phase angles as shown in Fig. 10. The frequency distribution of the phase angles shows that there are two groups of almost opposite phase1. This is explained by the fact that 8-day period is a standing wave over the Northern Hemisphere, and it is interesting that the amplitudes of the 8-day period in the action centers are large and the phases are almost the same.

Next, the amplitude and phase angles of 8-day period of the upper air temperature at Tateno are calculated. The results are shown in Fig. 11. Glancing at this figure, we see

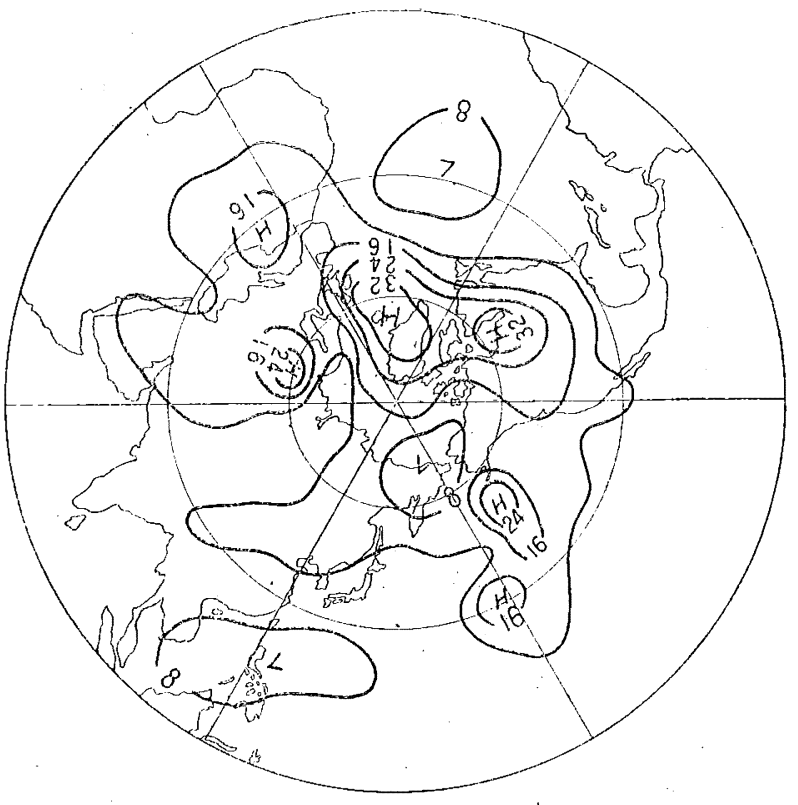

Fig. 8, Distribution of amplitude of 8-day period (500 mb) from Jan. 1 to Feb. 21949 in 10 feet. H:Large amplitude, L:Small amplitude

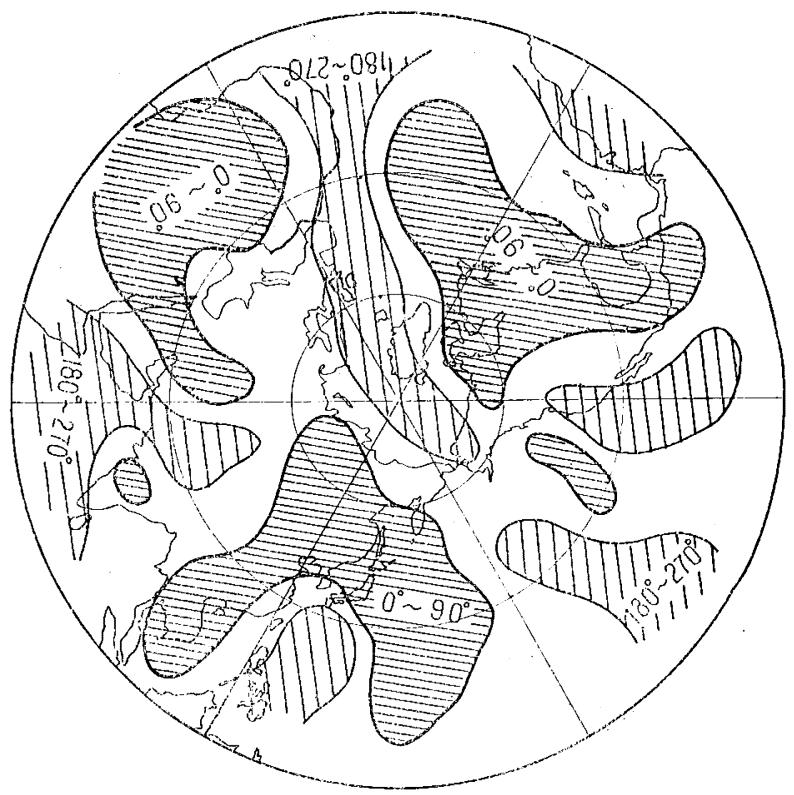

Fig. 9. Distribution of phase angle of 8-day period. $(500 \mathrm{mb})$ 


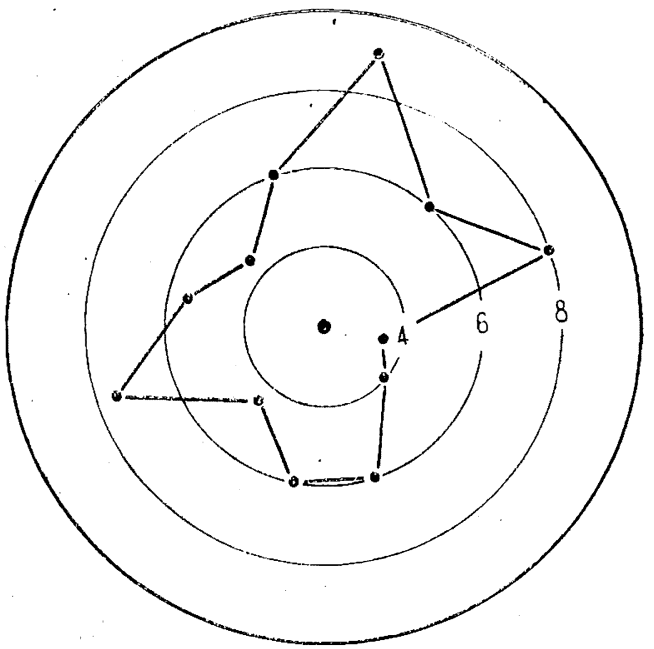

Fig. 10.

Frequency distribution of the phase angle of 8 -day period. (500mb)

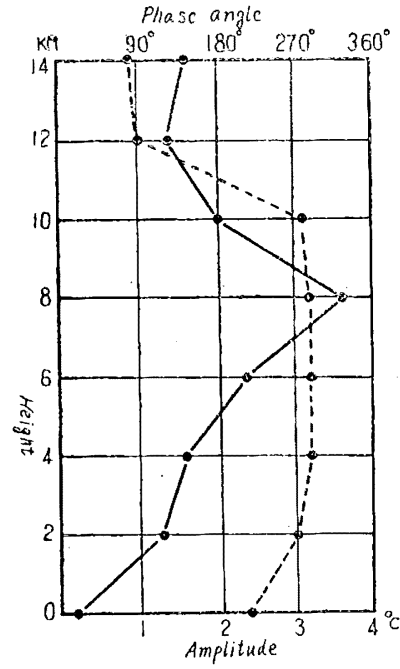

Fig. 11.

Height distribution of phase angle and amplitude of 8-day period of temperature from Nov. 30 to Dec. 311949 at Tateno. Full line represents the amplitude and dotted line the phase angle.

that the amplitude is quite large at about the tropopause and the phase of oscillation in the stratosphere is opposite to that in the troposphere. This is just the nature of the internal wave.

HAURWITZ [5] studied theoretically the oscillation of the atmosphere assuming two layers and concluded that several-day periodicity is possible, and K. TAKAHASHI [6] suggested that 7-day periodicity in winter is the internal oscillation of Siberian cold air mass. Therefore, it is quite probable that 7- or 8-day periodicity is the internal wave of the atmosphere over the Northern Hemisphere, though further researches are necessary to confirm it.

\section{4-day periodicity in weather}

Though the existence of 4-day periodicity in weather has been pointed out by many authors, definite explanation of the phenomena is not yet given. T. WATANABE studied such a periodicity.

Time variation of the latitude of isothermal line $10^{\circ} \mathrm{C}, 0600 \mathrm{LMT}$ January 1937 at meridian $140^{\circ} \mathrm{E}$ is shown in Fig. 12. From this figure, we see that (1) 4-day periodicity predominated at least during this period, (2) when 4-day periodical variation is disordered, a 
new 4-day periodical variation begins at that instant. Such nature is quite

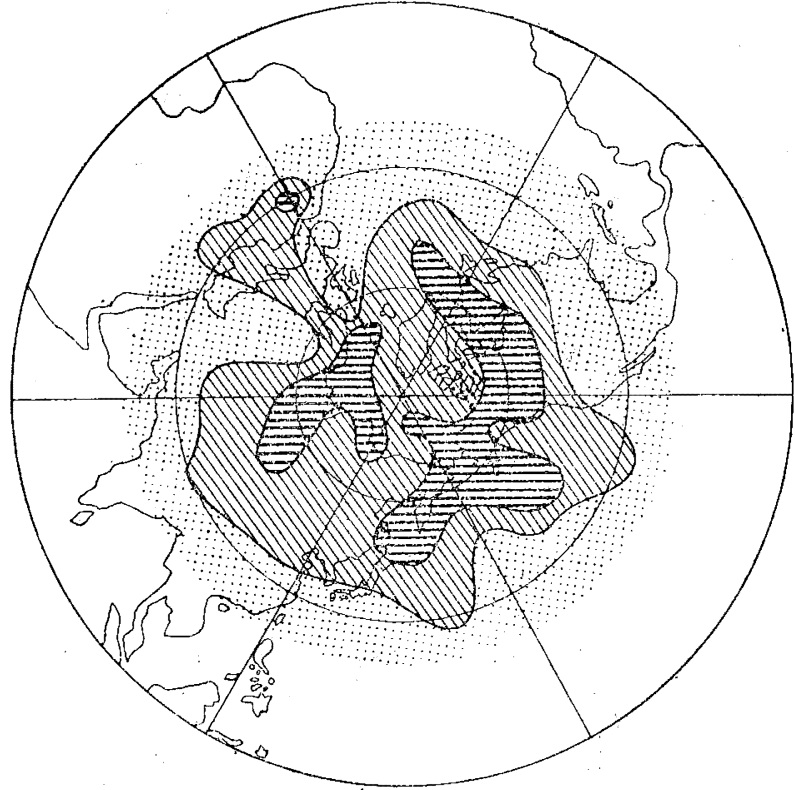

Fig. 13.

Distribution of the amplitude of the 4-day periodical variation of the height of $500 \mathrm{mb}$ surface in winter, 1949. (calculated in use of Histrical Weather Maps)

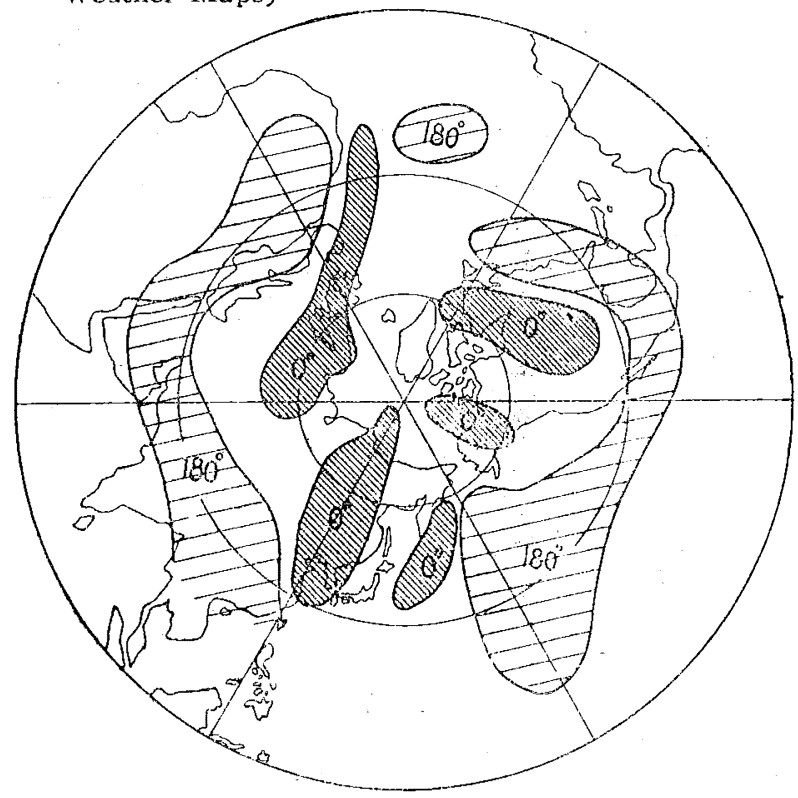

Fig. 14.

Distribution of the phase angle of the 4-day periodical variation of the height of $500 \mathrm{mb}$ surface in winter, 1949 common throughout the year, and they seem to be caused by cyclic passage of the upper trough, or westerly waves.

T. WATANABE analysed also $500 \mathrm{mb}$ height over the Northern Hemisphere during 1950 by periodogram analysis assuming 4-day period. The distribution of the amplitudes of 4-day period over the Northern Hemisphere is shown in Fig. 13. This distribution is correlated with the pattern of westerly flow, and it may be natural that we consider such an oscillation to be the wave motions of the zonal current. Further, the distribution of the phase angles is shown in Fig. 14. The pattern is not so simple as we expected at first. However, the change of phase angle at latitude $60^{\circ} \mathrm{N}$, shown in Fig. 15,

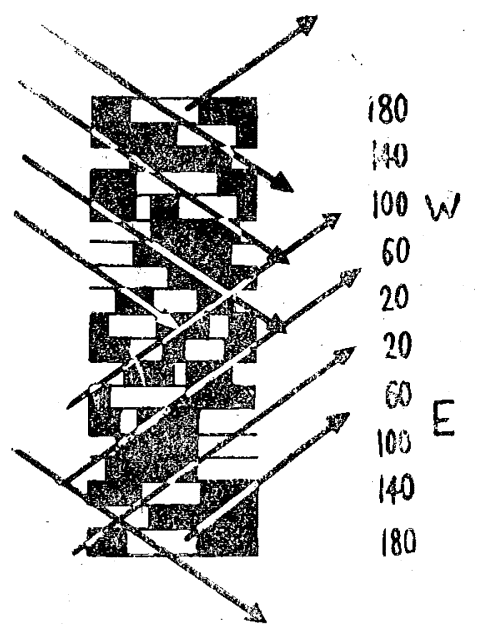

Fig. 15.

Longitudinal distribution on the phase (represented by black and white area) of the 4-day periodical variation at $60^{\circ} \mathrm{N}$, in winter. 


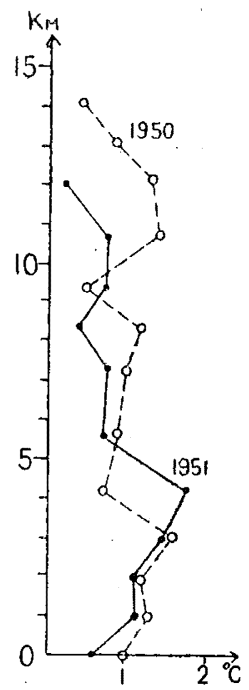

Fig. 16.

Vertical distribution of the amplitude of 4-day periodical change of upper temperature at Tateno, in winter, 1950 and 1951.

\section{Analysis of westerly} wave by wave length

We analysed the time variation of the pressure; and $\mathrm{S}$. KUBOTA analysed them by space variation of the pressure. Assuming that the pressure variations are built up of elementary waves of various wave lengths, he analysed westerly waves by harmonic analysis.

Namely, putting

$H(x, t)=$

$$
\left.\Sigma_{\{}^{\prime} A_{n}(t) \sin \left(2 n \pi x+\Psi_{n}\right)\right\}
$$

where $A_{n}, n, \Psi_{n}$ denote amplitude, wave number round the earth, and phase angle, respectively, and using the suggests that two types of waves exist, one moving towards the east with the speed of $40^{\circ}$ long./day and the other towards the west with the speed of $40^{\circ}$ long./day. If the two types of waves really exist, a standing wave is expected over the earth. This agrees qualitatively with the distribution of the phase angles in Fig. 14. We found 4 standing waves along the middle latitudes.

He investigated also the height distribution of the amplitudes and phases of 4-day period of the temperature at Tateno. The result is shown in Fig. 16. This is quite different from that of 8-day period, and it is doubtful that 4-day periodicity is caused by internal waves. Though T. WATANABE tried to explain them by the superposition of dispersive waves by following method adopted by CHARNEY-ELIASSEN for numerical prediction, the mechanism. of such a periodicity is not yet clear.

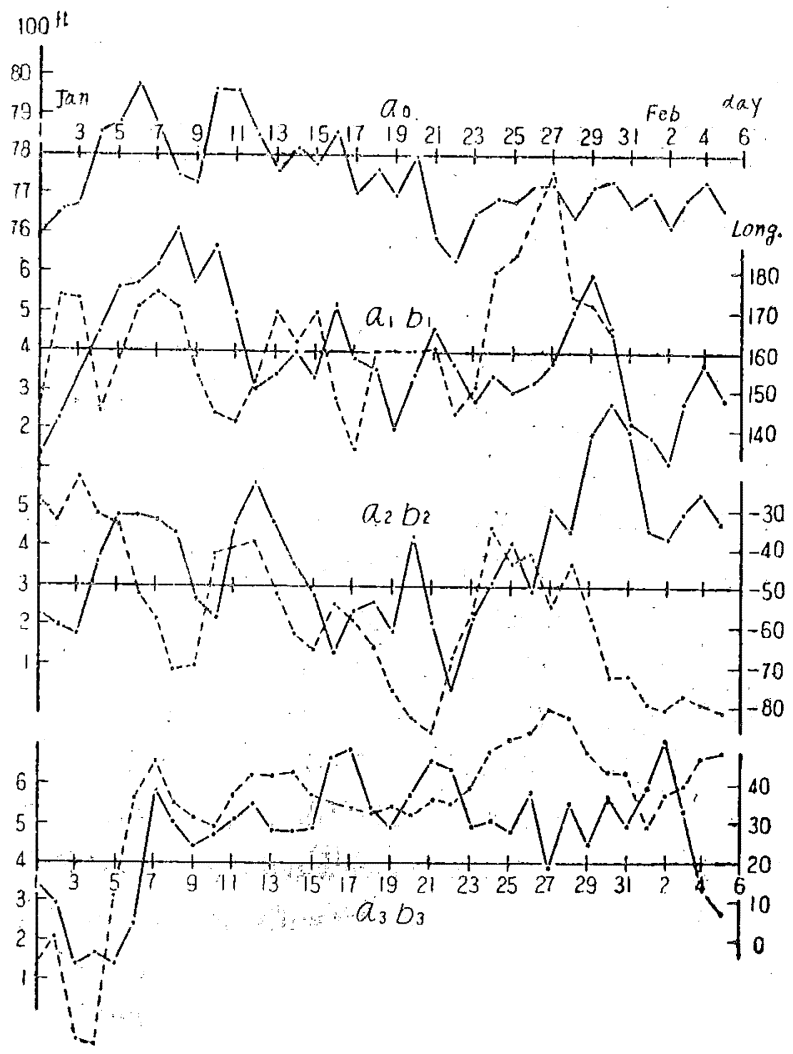

Fig. 17 (a).

Full line: Amplitude Broken line: Phase angle expressed by the position of trough 
estimated values at every 10 degrees in longitude, along $50^{\circ}$ $\mathrm{N}$-latitude circle on the $500 \mathrm{mb}$ isobaric surface on the Historical Weather Maps from the 1st; Jan. to the 5th, Feb. 1949, he obtained the time variation of amplitudes and phase-angles of component-waves as shown in Fig. 17 for $n=1,2,3,4,5$, and 6 respectively. The upper-most curve in these figures represents the mean height of the isobaric surface, full lines in other figures express amplitudes, and broken lines the phase angles of the componentwaves. In this case, the unit of the amplitudes is $100 \mathrm{ft}$, and the phases are expressed by the positions of the trough in longitude. Glancing at these figures, we find that (1) the periods of oscillations of waves,

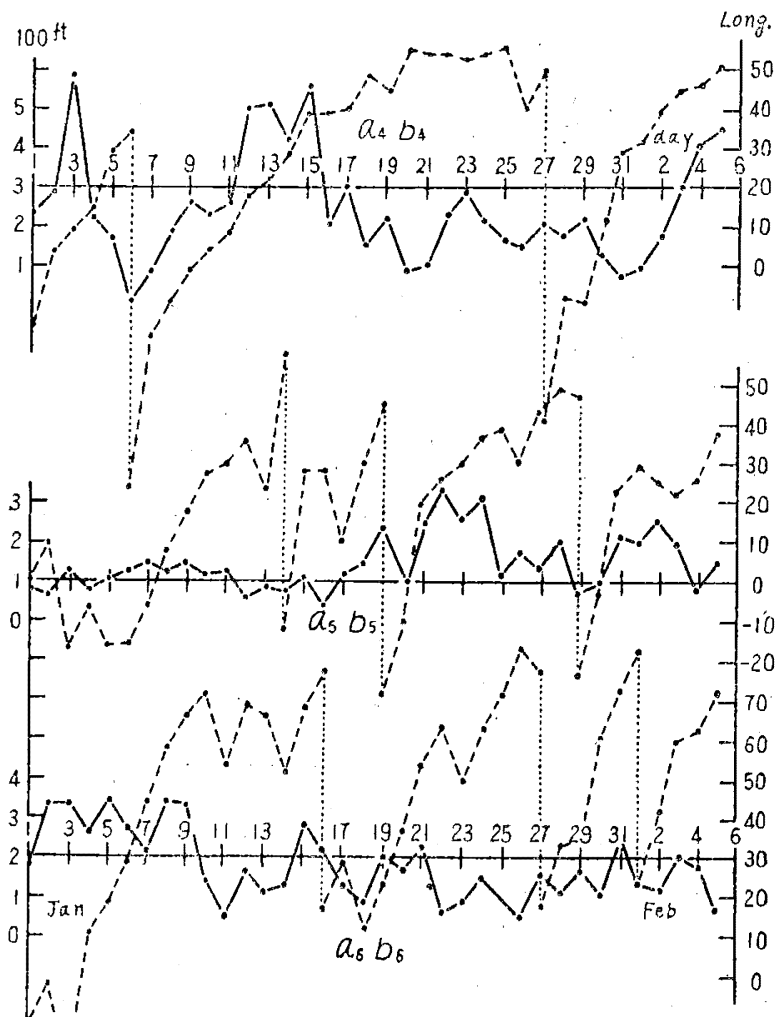

Fig. 17 (B). Full line: Amplitude Broken line: Phase angle expressed by the position of trough. whose wave number is 1,2 or 4 , are relatively long, while short periodic oscillations predominate in those, whose wave number is 3,5 or 6 , (2) the waves of wave number 1,2 or 3 seem to be oscillating around their mean positions, and their range of the oscillations is nearly $40^{\circ}$ in longitude and the wave of the wave number 3 was almost stationary from 7 th to $23 \mathrm{rd}$, Jan., (3) the difference between the westward and the eastward motion of wave number 1 is not noticeable, while the wave of wave number 2 progresses westward slowly at first and accelerates afterward and then after 7 or 8 days it suddenly retraces its course and returns to its former position in 2 or 3 days, continuing its progress further (Mean velocity of eastward motion is $12^{\circ}$ in long., that of the westward one is $6.8^{\circ}$ in long.), (4) the waves of wave-number 4 or more move eastward with decelerating speed and ultimately become almost stationary and after a while they commence discontinuously their eastward motion.

From these facts, it is clear that RossBY's formula concerning the propagation of the westerly trough in the middle latitudes does not hold even as to componentwaves. However, they suggest to us that there are two kinds of waves, one is a progressive wave and the other a standing one which may be attributed to topography. 
Accordingly, assuming that a component wave expressed by

$$
A_{n} \sin \frac{2 \pi}{L}\left(x+\phi_{n}\right)+B_{n} \sin \frac{2 \pi}{L}\left(x-C_{n} t+\Psi_{n}\right)
$$

where $L=2 \pi / n(n=1,2,3, \cdots \cdots), A_{n}, B_{n}$ and $C_{n}$ were calculated, and the relation was shown in Table 1. Of course, such a relation is not sufficient to express the actual data concretely, but we can grasp the general nature of the waves. In the above equation, the first term of the right hand side expresses the standing pressure pattern, while the second term expresses propagating waves. We see that velocity of the propagating waves depends on the wave number $n$. When $n=1,2$ the waves propagate towards the west, while when $n=4$, $5, \cdots \cdots$, they move towards

Table 1.

\begin{tabular}{l|rrrrrrrc}
\hline$n$ & 1 & 2 & 3 & 4 & 5 & 6 & 7 & unit \\
\hline$C_{n}$ & $-22^{\circ}$ & $-13^{\circ}$ & 0 & $4^{\circ}$ & $7^{\circ}$ & $9^{\circ}$ & $12^{\circ}$ & long./day \\
$D$ & $88^{\circ}$ & $93^{\circ}$ & $75^{\circ}$ & $76^{\circ}$ & $80^{\circ}$ & $77^{\circ}$ & $40^{\circ}$ & 1 at. \\
$A_{s}$ & 33 & 31 & 39 & 5 & 9 & 6 & 0 & $10 \mathrm{ft}$ \\
$A_{p}$ & 21 & 25 & 25 & 24 & 14 & 17 & 11 & $10 \mathrm{ft}$ \\
\hline
\end{tabular}

$n$ : wave number

$C_{n}$ : mean wave velocity

$D$ : width of disturbance

$A_{s}$ : amplitudes of stationary waves

$A_{p}$ : mean amplitudes of progressive wave

$U_{m}=16^{\circ}$ long./day (mean velocity of zonal current) the east. This agrees with that expected from Rossby's formula, but such an agreement is only qualitative.

\section{Extrapolation based on periodicities}

Periodicities have been applied often to seasonal forecasting already 100 years ago, or more. Y. NAKATA's method [8], which is one of the fundamental methods of the present seasonal forecasting in Japan, belongs to this category. M. OGAWARA [9] is carrying on researches about the method of auto-correlation. We proposed the method of extrapolation by periodgram analysis. Each of these methods has its own advantages, and among them, the auto-correlation method is founded on the most perspicuous theory. From the standpoint of practical application however, the method of harmonic analysis, which Prof. IMAHORI [10] has recently been discussing, is more advantageous.

Next, our extrapolation by harmonic analysis will be explained. Let $f(t)$ be the observed values of a certain meteorological element, such as pressure, temperature and so on. It is well known that the following relation holds,

$$
f(t)=\int_{0}^{\infty}\{\phi(\nu) \cos 2 \pi \nu t+\Psi(\nu) \sin 2 \pi \nu t\} d \nu
$$

where

$$
\phi(\nu)=2 \int_{-\infty}^{\infty} f\left(t^{\prime}\right) \cos 2 \pi \nu t^{\prime} d t^{\prime}, \quad \Psi(\nu)=2 \int_{-\infty}^{\infty} f\left(t^{\prime}\right) \sin 2 \pi \nu t^{\prime} d t^{\prime}
$$

under some conditions. This means that $f(l)$ is expressed as the sum of sinusoidal 
waves of various frequencies and $\phi(\nu), \psi(\nu)$ are spectrums for $\nu$. In reality, however, only the values of $f(t)$ in the past are known. Therefore we put, instead of (12),

$$
f(t)=\sum_{i} a_{i} \cos 2 \pi \nu_{i}+\sum_{i} b_{i} \sin 2 \pi \nu_{i} \mathrm{t}
$$

Assuming $a_{i}, b_{i}$ are independent on $t$ approximately, $a_{i}, b_{i}$ are calculated from the past values of $f(t)$ by harmonic analysis. Introducing such values into (14), the future values of $f(t)$ are calculated. This is the principle of the present method.

It must be noticed, however, that such a relation needs to be corroborated by actual data. We can not say apriori such an expression always holds. After researches on actual data, we found that it holds at least for long periodic variation of pressure and temperature.

Now $a_{i}, b_{i}$ are calculated from observed data during the time interval from 0 to $\tau$ by the following relations,

$$
\begin{aligned}
& a_{i}=\frac{2}{\tau} \int_{0}^{\tau} f(t) \cos 2 \pi \nu_{i} t d t=\frac{\mathcal{P}\left(\nu_{i}\right)}{\tau} \\
& b_{i}=\frac{2}{\tau} \int_{0}^{\tau} f(t) \sin 2 \pi \nu_{i} t d t=\frac{\psi\left(\nu_{i}\right)}{\tau} .
\end{aligned}
$$

Dividing the time interval of the integration of equation (12) into discrete periods of $1 / \tau$,

$$
f(t)=\int_{0}^{\infty}\left\{\mathcal{P}^{\prime}(\nu) \cos 2 \pi \nu t+\psi\left(\nu^{\prime \prime}\right) 2 \pi \nu t\right\} d \nu=\left\{\sum_{i} \rho\left(\nu_{i}\right) \cos 2 \pi_{i} t+\sum_{i} \psi\left(\nu_{i}\right) \sin 2 \pi \nu_{i} t\right\} \frac{1}{\tau}
$$

where $\nu_{i+1}-\nu_{i}=\frac{1}{\tau}$ and $\phi(\nu), \psi(\nu)$ are slowly varying functions of $\nu$. This expresses that continuous spectrums $\left.\phi(\nu), \psi^{\prime} \nu\right)$ are replaced by discrete line spectrums. Such a transformation produces uncertainty of the magnitude about $1 / \tau$ in $\nu$, and if $\nu$ has errors of $\delta \nu$, relative errors of $2 \sin \delta \nu_{i} t$ will be produced in future values of the elementary waves.

Accordingly,

$$
t \leqq \frac{\varepsilon}{2 \pi \delta \nu_{i}}, \quad \text { or } \quad t \leqq \frac{\tau \varepsilon}{2 \pi} .
$$

That is to say, the forecast periods depend on the periods of analysed time interval $\tau$, and the allowable errors of the forecast. It is also concluded that the number of waves which are used for the analysis depends on the time interval of the analysis.

\section{Application to actual seasonal forecasting}

Our method was applied to seasonal forecasting of 5-day mean pressure and 5day mean temperature assuming that 25-, 35-, 45- and 65-day period existed in their time variation. An example of the numerical calculation is shown in Table 2. 


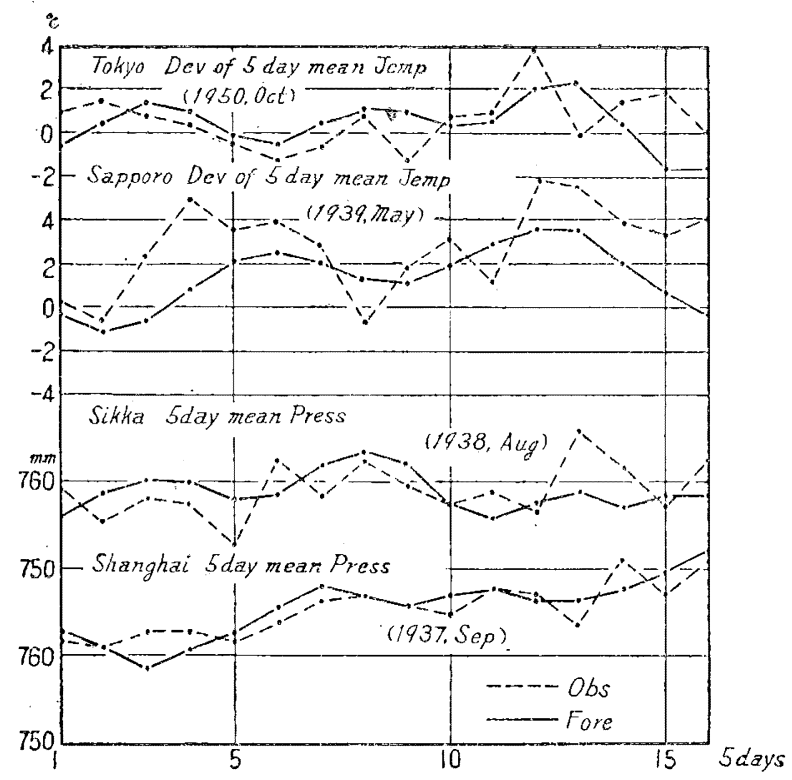

Fig. 18. Examples of forecast, full lines represent forecasted values and dottel line observed.

After such calculations of many test cases, we found that forecasted values agree in general tendency within 2 months. In Fig. 18 some examples are shown, where full lines are forecasted values, dotted lines are observed values. Agreement between the two was tested by taking correlation coefficients between them, and we found that the correlation coefficients were always positive and about 0.4. Of course, the deviations from the normal are significant in the present method, hence for obtaining 5-day mean pressure, we must add the normal 5-day mean values to the calculated values. Thus, though the numerical agreements are not yet enough for precise forecast, our method encourages us in ropes of, at least, qualitative forecasting, especially for temperature forecasting.

We tried also to make prognositic charts of 5-day mean pressure deviation from the normal, but the results are not yet satisfactory and further investigation is wanted. Acknowledgment - we wish to express our sincere thanks to Director $\mathrm{H}$. HATAKEYAMA for his encouragement to our researches and to Dr. H. ARAKAWA for his valuable advice.

\section{References}

[1] Tаканashi, K. and Collaborators, 19j0: Studies on the Seasonal Weather Forecasting, Papers in Meteorology and Geophysics 2, p. 6.

[2] Werckman, L. 1929: Die Termische Wirkung der 24 Tägligen Problem Wellen des Winters 1923-1924, Beiträge zur Physik der Freien Atmesphäere Hergesell Heltband.

[3 ] LET'Nu, H. 1931: Theoretische Ableitung und Physikalishen Nachweiss einer 36 Tägligen Luftdruck Wellen, Veröff. Geophys. Inst. Leipzig, 20.

[4] Watker, G. 1940. On Periods and Symmetry Points in pressure as Aids to Forecasting, Quarterly Journal of the Royal Meteorological Society. 72, p. 265.

[5] Haurwry, B. 1937: The Oscillation of the Atmosphere, Beit. z. Geophy. 5i, p. 195.

[6] Takninasirr, K. 1940: Dynamic Climatorogical Consideration on the Alternation of Weather 
in Early Winter in the Far East, Journal of the Meteorological Society of Japan. 18, p. 177.

[7] Channey, and Eliassen, 1949: A Numerical Method for Prediction of the Perturbation of the Middle Latitude Westerlies. Tellus, 1, p. 38.

[8] Nakata, Y. 1950: Long-range Forecasting by Periodicity Extrapolation Method. Geographical Magazin 22, p. 99.

[9] OGawara, M. 1947: Forecasting Method by Stochastic Extrapolation, Temporary Report of Central Meteorological Observatory, 24, p. 1.

[10] Imahori, K. and Kobayasiin, T. 1951: On the Long-rane Period Forecasting by Means of Harmonic Analysis, Journal of the Meteorological Society of Japan 29 p. 365. 